\title{
Continuously tunable narrowband pulses in the THz gap from laser-modulated electron bunches in a storage ring
}

\author{
P. Ungelenk, ${ }^{*}$ M. Höner, H. Huck, ${ }^{\dagger}$ S. Khan, C. Mai, and A. Meyer auf der Heide \\ Center for Synchrotron Radiation (DELTA), TU Dortmund University, D-44227 Dortmund, Germany \\ C. Evain, C. Szwaj, and S. Bielawski \\ Laboratoire de Physique des Lasers, Atomes et Molécules (PhLAM), \\ Centre d'Études et de Recherches Lasers et Applications (CERLA), \\ Université des Sciences et Technologies de Lille, \\ F-59655 Villeneuve d'Ascq Cedex, France
}

(Received 27 September 2016; published 21 February 2017)

\begin{abstract}
This article reports on the generation of narrowband coherent synchrotron radiation from an electron storage ring. For the first time, this kind of radiation was now produced with continuously tunable frequencies in the so-called "THz gap" (between 1.2 and $5.6 \mathrm{THz}$ ), whereas previous experiments were limited to below $750 \mathrm{GHz}$. The experiment was performed at the DELTA storage ring in Dortmund, Germany, employing the interaction of external intensity-modulated laser pulses with an electron bunch, which causes a periodic longitudinal modulation of the charge density on a sub-millimeter scale. Furthermore, a strong influence of third-order dispersion in the laser pulses on the bandwidth and peak intensity of the $\mathrm{THz}$ radiation was observed. This effect is discussed in detail based on numerical simulations of the laser pulse generation and laser-electron interaction, and a modification of the laser system for compensating third-order dispersion is proposed.
\end{abstract}

DOI: 10.1103/PhysRevAccelBeams.20.020706

\section{INTRODUCTION}

Coherent synchrotron radiation (CSR) pulses in the terahertz $(\mathrm{THz})$ regime have become of increasing interest for the study of beam dynamics and instabilities in electron storage rings (e.g., see $[1,2]$ ) as well as for a growing number of user applications (e.g., see [3-6]), since the power of CSR exceeds the power of incoherent synchrotron radiation by several orders of magnitude. CSR in the THz regime occurs when sub-picosecond structures are present in the longitudinal electron distribution, allowing the electromagnetic waves generated by single electrons to add up constructively at sub-millimeter wavelengths.

At electron storage rings, broadband CSR pulses in the $\mathrm{THz}$ regime were first observed as spontaneous radiation bursts (e.g., see [7-10]) due to the microbunching instability $[11,12]$. When the bunch length is reduced to a few picoseconds, e.g., during low- $\alpha$ operation, stable broadband CSR emission in the $100-\mathrm{GHz}$ regime is achieved (e.g., see [13]).

\footnotetext{
"peter.ungelenk@tu-dortmund.de

now at Deutsches Elektronen-Synchrotron DESY, Zeuthen location, D-15738 Zeuthen, Germany.

Published by the American Physical Society under the terms of the Creative Commons Attribution 4.0 International license. Further distribution of this work must maintain attribution to the author(s) and the published article's title, journal citation, and DOI.
}

Stable THz CSR can also be obtained by seeding the electron bunches with an external laser. In recent years, several facilities are employing a laser-electron interaction in an undulator to imprint an energy modulation onto a short central slice of a longer electron bunch (e.g., see [14-19]). The main goal is to generate ultrashort pulses at short wavelengths in the X-ray or vacuum-ultraviolet (VUV) regimes based on the femtoslicing [20] or coherent harmonic generation (CHG) [21,22] techniques. Energy-dependent path length differences in the magnet lattice of the storage ring then cause the off-energy electrons to vacate the central slice. This leads to a sub-picosecond dip in the longitudinal electron density giving rise to broadband multi-THz CSR (e.g., see [23-26]). With the dip broadening and slowly disappearing from turn to turn, decreasing CSR emission in the sub-THz regime has been observed for several revolutions in the storage ring after the initial laser-electron interaction (e.g., see [27,28]).

Early on, it was proposed to manipulate the laser pulse shape in order to generate tailored CSR pulses, e.g., to employ an equidistant laser pulse train resulting in a periodic electron density modulation and a narrowband CSR spectrum [24]. First successful experiments of generating continuously tunable narrowband laserinduced CSR in the sub- $\mathrm{THz}$ regime were performed at the UVSOR storage ring in Okazaki, Japan, using a long intensity-modulated laser pulse [29]. The modulated laser pulse was generated based on the chirped-pulse-beating 
technique [30,31], at which a long chirped laser pulse is sent through a Michelson interferometer. Recombining the two chirped laser pulse copies with a small delay leads to a beating effect, and the frequency of the intensity modulation (and, thus, of the CSR) can easily be tuned by the variable length of one interferometer arm. Requiring only a few additional optical components in the laser beamline, this technique can be adopted with little effort by most facilities which already employ a laser-electron interaction based on a short-pulse laser system. The tuning range of the CSR generation based on chirped-pulse beating is eventually limited by the dispersive properties of the storage ring lattice, leading to a range of about $75 \mathrm{GHz}$ to $750 \mathrm{GHz}$ in the case of UVSOR [32].

This article reports on the generation of narrowband CSR pulses at the electron storage ring DELTA in Dortmund, Germany. For the first time, continuously tunable frequencies between 1.2 and $5.6 \mathrm{THz}$ were achieved with a bandwidth ranging from 190 to $340 \mathrm{GHz}$, a repetition rate of $1 \mathrm{kHz}$, and a pulse energy of 0.1 to $1 \mathrm{~nJ}$. Furthermore, the effect of a nonlinear chirp in the laser pulse on the intensity and spectral shape of the CSR pulses is investigated and a modification of the laser system is proposed in order to counteract this effect.

Section II explains the principle of generating narrowband CSR including a review of chirped-pulse beating, the emission of CSR, the laser-electron interaction, the evolution of the longitudinal electron density, and the effect of third-order dispersion. Section III presents numerical calculations for the tuning range of the narrowband $\mathrm{THz}$ radiation and the effect of third-order dispersion as well as a start-to-end simulation. The experimental setup used at the electron storage ring DELTA is described in Sec. IV. Finally, the generated narrowband $\mathrm{THz}$ spectra are shown and discussed in Sec. V.

\section{GENERATION OF NARROWBAND CSR BASED ON CHIRPED-PULSE BEATING}

\section{A. Basic principle}

The general layout of the experiment is presented in Fig. 1(a). A Ti:sapphire short-pulse laser system based on chirped-pulse amplification [33] is employed for most of the laser-seeding schemes. Here, an ultrashort pulse from a laser oscillator (top left) is temporally stretched before entering the amplifier gain medium. Following amplification, the chirped laser pulse (colored waveform) passes the internal compressor of the amplifier, which-in case of chirped-pulse beating - is detuned to retain a nonzero chirp. A Michelson interferometer is used to split and then recombine the pulse with a variable delay, which results in an intensity modulation with tunable frequency in the $\mathrm{GHz}$ or $\mathrm{THz}$ regime. Inside an undulator (called "modulator"), the intensity-modulated laser pulse imprints a periodic energy modulation with a (sub-) millimeter amplitude modulation onto an electron bunch, and this energy modulation is converted into a (sub-) millimeter density modulation by the subsequent dispersive magnet lattice. Corresponding to the Fourier transform of the density modulation, a narrowband CSR pulse in the $100-\mathrm{GHz}$ or $\mathrm{THz}$ regime is emitted at a bending magnet further downstream.

As an illustration and a main result of this article, a modulated laser pulse shape (autocorrelator trace) and the corresponding narrowband $\mathrm{THz}$ spectrum centered at $3 \mathrm{THz}$ are presented in Fig. 1(b,c).

\section{B. Chirped-pulse beating}

A laser pulse with central frequency $\omega_{0}$ propagating through an arbitrary dispersive optical setup is subject to a non-linear frequency-dependent phase advance $\varphi(\omega)$, which can be expanded into a Taylor series

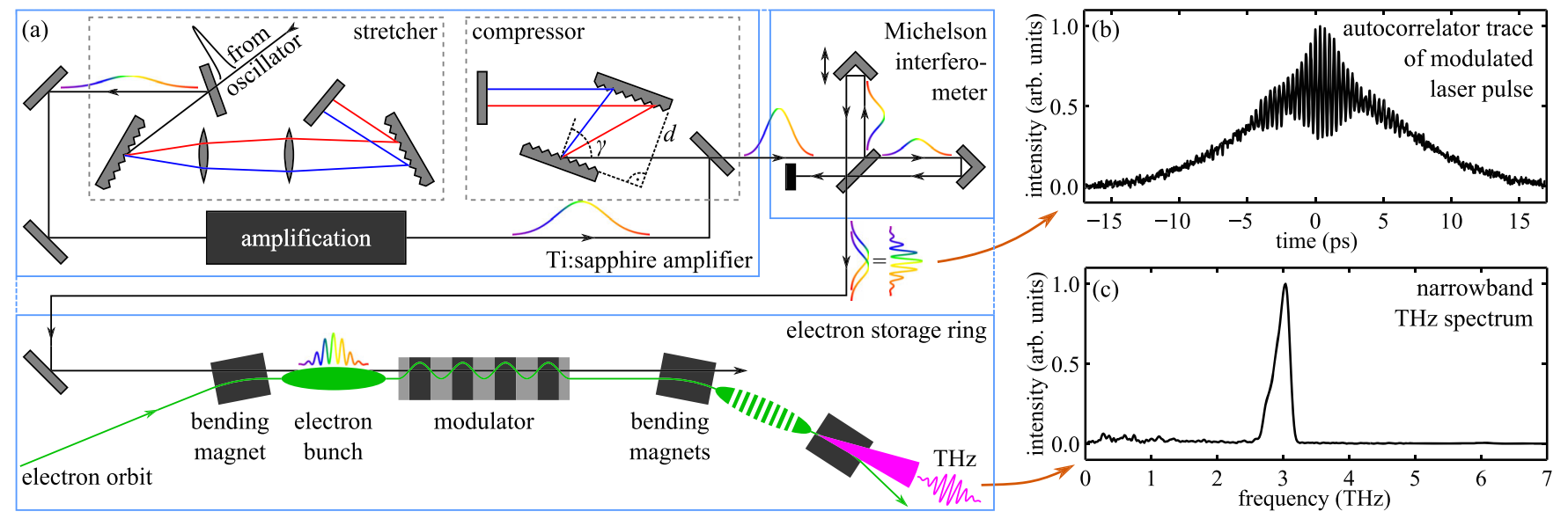

FIG. 1. Sketch of the chirped-pulse-beating setup used for the generation of narrowband laser-induced CSR at the electron storage ring DELTA in Dortmund, Germany, (a) and examples of a recorded laser pulse shape (b) and the corresponding narrowband $\mathrm{THz}$ spectrum (c). 


$$
\begin{aligned}
\varphi(\omega)= & D_{0}+D_{1} \cdot\left(\omega-\omega_{0}\right)+D_{2} \cdot\left(\omega-\omega_{0}\right)^{2} \\
& +D_{3} \cdot\left(\omega-\omega_{0}\right)^{3}+\cdots .
\end{aligned}
$$

Here, $D_{0}=\varphi\left(\omega_{0}\right)$ is the central phase advance, $D_{1}$ is the group delay, $D_{2}$ is the group delay dispersion (GDD), and $D_{3}$ is a cubic phase modulation (all evaluated at $\omega_{0}$ ).

At full compression, the laser system is assumed to generate a Fourier-limited or bandwidth-limited laser pulse (i.e., zero chirp) with an electric field

$$
E_{0}(t)=\mathcal{E} \cdot e^{-a_{0} t^{2}} \cdot e^{i \omega_{0} t}
$$

and amplitude $\mathcal{E}$. The parameter $a_{0}=2 \ln 2 / \Delta t_{0}^{2}$ is an inverse measure of the pulse length $\Delta t_{0}$ (FWHM), which is given by the time-bandwidth product $\Delta t_{0} \cdot \Delta \nu=2 \ln 2 / \pi$ for a Gaussian-shaped pulse with spectral width $\Delta \nu$ (FWHM). Taking dispersion into account only up to the second order (the GDD $D_{2}$ ), the electric field of a chirped pulse exiting a detuned compressor is given by

$$
E_{1}(t)=\mathcal{E} \sqrt{\frac{\Delta t_{0}}{\Delta t_{1}}} \cdot e^{-a_{1} t^{2}} \cdot e^{i \omega_{0} t+i b_{1} t^{2}},
$$

with $\sqrt{\Delta t_{0} / \Delta t_{1}}$ accounting for the lower peak intensity of the elongated pulse and $a_{1}=2 \ln 2 / \Delta t_{1}^{2}$. Following the work of Weling and Auston [31] and assuming a fixed bandwidth $\Delta \nu$, the increased pulse length

$$
\Delta t_{1}=\Delta t_{0} \sqrt{1+\left(\frac{4 D_{2} \ln 2}{\Delta t_{0}^{2}}\right)^{2}}
$$

allows for the chirp parameter to be approximated by

$$
\left|b_{1}\right| \approx \frac{2 \ln 2}{\Delta t_{1} \Delta t_{0}}
$$

for $\Delta t_{1} \gg \Delta t_{0}$. In Eq. (3), the zero- and first-order dispersion have not been included, as they do not affect the pulse shape or length. Introducing a delay $\tau$ within the Michelson interferometer, the intensity of the recombined pulse is given by

$$
\begin{aligned}
I \propto & \frac{1}{2}\left|E_{1}\left(t+\frac{\tau}{2}\right)+E_{1}\left(t-\frac{\tau}{2}\right)\right|^{2} \\
= & \frac{\mathcal{E}^{2}}{2} \frac{\Delta t_{0}}{\Delta t_{1}}\left[e^{-2 a_{1}(t+\tau / 2)^{2}}+e^{-2 a_{1}(t-\tau / 2)^{2}}\right. \\
& \left.+2 e^{-2 a_{1}\left(t^{2}+\tau^{2} / 4\right)} \cdot \cos \left(\omega_{0} \tau+2 b_{1} \tau t\right)\right] .
\end{aligned}
$$

The overall pulse shape in Eq. (7) is determined by the sum of two Gaussian-shaped pulses shifted by $\pm \tau / 2$, whereas the third term represents the intensity modulation with frequency

$$
f_{\mathrm{m}}=\frac{\left|b_{1}\right| \tau}{\pi}=\frac{2 \ln 2 \cdot \tau}{\pi \Delta t_{1} \Delta t_{0}} \approx \frac{\tau}{2 \pi\left|D_{2}\right|}
$$

and bandwidth (FWHM)

$$
\Delta f_{\mathrm{m}}=\frac{2}{\pi} \sqrt{2 \ln 2 \cdot a_{1}}=2 \Delta \nu \frac{\Delta t_{0}}{\Delta t_{1}} \approx \frac{\Delta t_{0}}{\pi\left|D_{2}\right|},
$$

which is two times the optical bandwidth of the laser scaled down by the stretching ratio $\Delta t_{0} / \Delta t_{1}$. The amplitude of the intensity modulation drops with $\tau$ as $\exp \left(-a_{1} \tau^{2} / 2\right)$, leading to a tuning range (FWHM) of

$$
\begin{gathered}
\Delta \tau=\Delta t_{1} \quad \text { or } \\
\Delta f_{\mathrm{m}}^{\Delta \tau}=\frac{2 \ln 2 \cdot \Delta \tau}{\pi \Delta t_{1} \Delta t_{0}}=\Delta \nu,
\end{gathered}
$$

which is identical to the optical bandwidth of the laser. However, the tuning range for the generation of narrowband CSR is limited by dispersive properties of the storage ring lattice (see Sec. II D).

\section{Emission of coherent synchrotron radiation}

With the single-electron power spectrum $P_{\mathrm{e}}(\omega)$ and the number of electrons $N_{\mathrm{e}}$ in a bunch, the total emission spectrum of an electron bunch is given by $[34,35]$

$$
P(\omega)=P_{\mathrm{e}}(\omega) N_{\mathrm{e}}\left[1+\left(N_{\mathrm{e}}-1\right) g^{2}(\omega)\right] .
$$

The so-called form factor

$$
g^{2}(\omega)=\left|\int \rho(t) e^{i \omega t} \mathrm{~d} t\right|^{2}
$$

which is the squared Fourier transform of the longitudinal electron density $\rho(t)$, determines the coherent emission spectrum proportional to $N_{\mathrm{e}}\left(N_{\mathrm{e}}-1\right) \approx N_{\mathrm{e}}^{2}$.

\section{Laser-electron interaction and evolution of the electron density}

The charge density of an electron bunch in a storage ring before the laser-electron interaction may be described by [35]

$$
\rho(\mathbf{R})=\rho_{0} \cdot \exp \left[-\frac{1}{2}\left(\frac{x^{2}}{\sigma_{x}^{2}}+\frac{x^{\prime 2}}{\sigma_{x^{\prime}}^{2}}+\frac{z^{2}}{\sigma_{z}^{2}}+\frac{\Delta E^{2}}{\sigma_{E}^{2}}\right)\right],
$$

where $\mathbf{R}=\left(x, x^{\prime}, z, \Delta E\right)$. The coordinates $x$ and $z$ are the horizontal and longitudinal electron position relative to the center of the bunch, $x^{\prime}$ is the horizontal angle of the particle trajectory, $\Delta E$ is the deviation from the central beam energy $E, \sigma_{x}$ and $\sigma_{x^{\prime}}$ are the horizontal rms bunch size and divergence, $\sigma_{z}$ is the rms bunch length, $\sigma_{E}$ is 
the natural rms energy spread, and $\rho_{0}$ is the peak density. The dependence on the vertical position $y$ and angle $y^{\prime}$ is neglected as it has no influence on the following considerations.

Assuming a sinusoidal transverse electron trajectory in an undulator tuned to the wavelength $\lambda$ of a collinearly propagating plane-wave laser pulse, the electron energy changes according to $\Delta W(z)=A(z) \sin (2 \pi z / \lambda)$, where $A(z)$ is the amplitude of the energy modulation, which is proportional to the laser field amplitude. This leads to a modified charge density

$\rho_{\mathrm{m}}(\mathbf{R})=\rho_{0} \cdot \exp \left[-\frac{1}{2}\left(\frac{x^{2}}{\sigma_{x}^{2}}+\frac{x^{\prime 2}}{\sigma_{x^{\prime}}^{2}}+\frac{z^{2}}{\sigma_{z}^{2}}+\frac{\Delta E_{\mathrm{m}}(z)^{2}}{\sigma_{E}^{2}}\right)\right]$

with $\Delta E_{\mathrm{m}}(z)=\Delta E-\Delta W(z)$.

With the transfer matrix of linear beam optics for the horizontal and longitudinal planes [35]

$$
\mathbf{T}=\left(\begin{array}{cccc}
r_{11} & r_{12} & 0 & r_{16} \\
r_{21} & r_{22} & 0 & r_{26} \\
r_{51} & r_{52} & 1 & r_{56} \\
0 & 0 & 0 & 1
\end{array}\right)
$$

describing the lattice section between laser-electron interaction and source point of the $\mathrm{THz}$ radiation, the charge density at the source point is given by [32]

$$
\tilde{\rho}_{\mathrm{m}}(\mathbf{R})=|\mathbf{T}|^{-1} \cdot \rho_{\mathrm{m}}\left(\mathbf{T}^{-1} \cdot \mathbf{R}\right) .
$$

Further following Evain et al. [32], a set of approximations is introduced in order to find an analytical expression for the form factor $g(\nu)$ [see Eq. (13)]: (i) The longitudinal laser field amplitude and the corresponding energy modulation amplitude $A(z)$ vary slowly compared to the oscillation $\sin (2 \pi z / \lambda)$ of the electrical laser field (slowlyvarying-envelope approximation). (ii) The finite electron bunch length and laser pulse duration are neglected. (iii) The chirped-pulse-beating technique is applied to the laser pulse, leading to an energy modulation amplitude of $A(z)=A_{0} \cos \left(2 \pi f_{\mathrm{m}} z / c\right)$. (iv) The laser intensity and the corresponding energy modulation amplitude $A_{0}$ are small so that $r_{56} \cdot A_{0} / E \ll \lambda_{\mathrm{m}} /(2 \pi)$, where $E$ is the nominal electron beam energy. In other words, the longitudinal displacement $r_{56} \cdot A_{0} / E$ of electrons with maximum energy modulation is much smaller than the inverse wave number $\lambda_{\mathrm{m}} /(2 \pi)$ of the laser amplitude modulation. Based on these approximations, a Fourier transform of the charge density $\tilde{\rho}_{\mathrm{m}}(\mathbf{R})$ leads to the so-called gain curve [32]

$$
\begin{aligned}
g^{2}\left(f_{\mathrm{m}}\right) \propto & \left(r_{56} \frac{\sigma_{E}}{E}\right)^{4}\left(\frac{2 \pi f_{\mathrm{m}}}{c}\right)^{4} \\
& \cdot \exp \left[-\left(\frac{2 \pi f_{\mathrm{m}}}{c}\right)^{2}\left(r_{51}^{2} \sigma_{x}^{2}+r_{52}^{2} \sigma_{x^{\prime}}^{2}+r_{56}^{2} \frac{\sigma_{E}^{2}}{E^{2}}\right)\right] .
\end{aligned}
$$

This gain curve is the envelope of all possible form factors with individual modulation frequency $f_{\mathrm{m}}$ and depends only on parameters of the storage ring optics. The shape $g(\nu)$ of a single narrowband $\mathrm{THz}$ spectrum is determined by the actual shape and finite longitudinal dimension of the laser pulse and its intensity modulation.

\section{E. Third-order dispersion}

According to [36], the grating compressor depicted in Fig. 1(a) introduces a GDD

$$
D_{2}=-2 \cdot \frac{4 \pi^{2} c d}{\omega_{0}^{3} g^{2}}\left\{1-\left[\frac{2 \pi c}{\omega_{0} g}-\sin \gamma\right]^{2}\right\}^{-\frac{3}{2}},
$$

where $d$ is the grating separation, $g$ is the grating constant (distance between lines), $\gamma$ is the angle of incidence, and $c$ is the speed of light.

Due to the nonlinear dependence of the beam path length on the wavelength inside the compressor, also a third-order dispersion is introduced [37]

$$
D_{3}=-\frac{D_{2}}{2 \omega_{0}} \cdot \frac{1+\frac{2 \pi c}{\omega_{0} g} \sin \gamma-\sin ^{2} \gamma}{1-\left[\frac{2 \pi c}{\omega_{0} g}-\sin \gamma\right]^{2}},
$$

which results in a quadratic chirp of the outgoing pulse [31]

$$
E_{1}(t) \propto e^{i \alpha t^{3}}
$$

and a linear chirp of the intensity modulation [31]

$$
I \propto \cos \left(2 \pi f_{\mathrm{m}} t+3 \alpha \tau t^{2}\right),
$$

where $\alpha\left(\Delta \nu, D_{3}\right)$ is a cubic phase coefficient depending on the third-order dispersion $D_{3}$ and the optical bandwidth of the laser $\Delta \nu$. This chirp of the intensity modulation implies an asymptotically linear increase of the modulation bandwidth with $\alpha$ and $\tau$ [31], which decreases the peak intensity of narrowband CSR spectra.

\section{NUMERICAL CALCULATIONS}

\section{A. Gain curve for the setup at DELTA}

Figure 2 shows the gain curve for the short-pulse facility at DELTA (see Sec. IV A) calculated according to Eq. (18) using the bunch properties $\left(\sigma_{x}, \sigma_{x^{\prime}}\right.$, and $\left.\sigma_{E} / E\right)$ at the modulator and the elements of the transport matrix from the modulator to the $\mathrm{THz}$ beamline ("turn 0"). A multiplication 


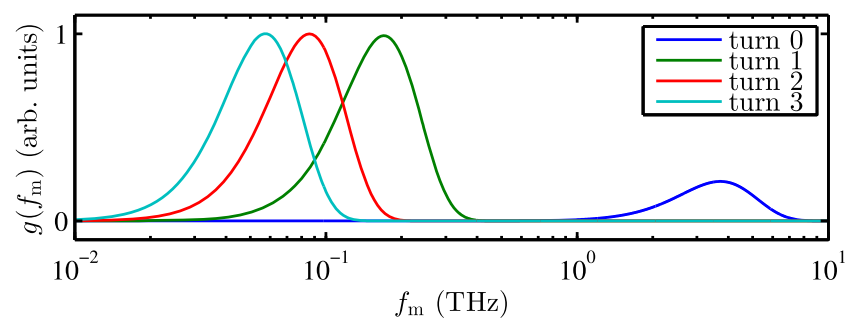

FIG. 2. THz gain curve for the short-pulse facility at DELTA according to Eq. (18) for turn 0 to 3 (see text for details).

of this turn- 0 transport matrix with the one-turn matrix starting at the $\mathrm{THz}$ beamline leads to a new set of transport matrix elements, which have been used for calculating the gain curves after one or more revolutions of the modulated electron bunch in the storage ring ("turn 1", "turn 2", "turn 3"). The bunch properties and matrix elements have been extracted from a numerical simulation of the storage ring lattice with the code ELEGANT [38], and a selection of parameters is given in Table I. As a result, the chirpedpulse-beating technique can be employed at DELTA to generate coherent narrowband $\mathrm{THz}$ radiation with a tuning range of about 1 to $7 \mathrm{THz}$ for the signal directly after the laser-electron interaction, or with a tuning range of about 10 to $300 \mathrm{GHz}$ for the signal after one or more revolutions.

\section{B. Effect and compensation of third-order dispersion}

The effect of third-order dispersion on the chirped-pulsebeating technique has been studied using an in-house numerical simulation code, which starts with a Fourierlimited pulse in time domain [Eq. (2)]. A series of fast Fourier transforms is then applied to perform the phase modulation [Eq. (1)] in frequency domain, the propagation through the Michelson interferometer in time domain, and a final calculation of the modulation frequency and bandwidth in frequency domain.

Figure 3 shows the analytical dependence of $f_{\mathrm{m}}$ and $\Delta f_{\mathrm{m}}$ on the interferometer delay $\tau$ based on Eqs. (8) and (9), which only include second-order dispersion $\left(D_{3}=0\right)$. The numerical results are extracted from the simulation. The parameters used for the analytical calculation as well as the numerical simulation are $\lambda_{0}=800 \mathrm{~nm}, \Delta t_{0}=40 \mathrm{fs}$, $\Delta t_{1}=11 \mathrm{ps}$ (value chosen for the experimental study,

TABLE I. Parameters for the calculation of the turn-0 to turn-3 gain curves for DELTA (see text for details).

\begin{tabular}{lcccc}
\hline \hline & turn 0 & turn 1 & turn 2 & turn 3 \\
\hline$r_{51}\left(10^{-2}\right)$ & -4.92 & -9.88 & -2.25 & 8.26 \\
$r_{52}(\mathrm{~mm} / \mathrm{rad})$ & 15.2 & -147 & -235 & -137 \\
$r_{56}(\mathrm{~mm})$ & 18.1 & 581 & 1154 & 1728 \\
$\sigma_{x}(\mu \mathrm{m})$ & \multicolumn{5}{c}{270} \\
$\sigma_{x^{\prime}}(\mu \mathrm{m} \mathrm{rad})$ & \multicolumn{5}{c}{103} \\
$\sigma_{E} / E$ & $6.8 \times 10^{-4}$ \\
\hline \hline
\end{tabular}
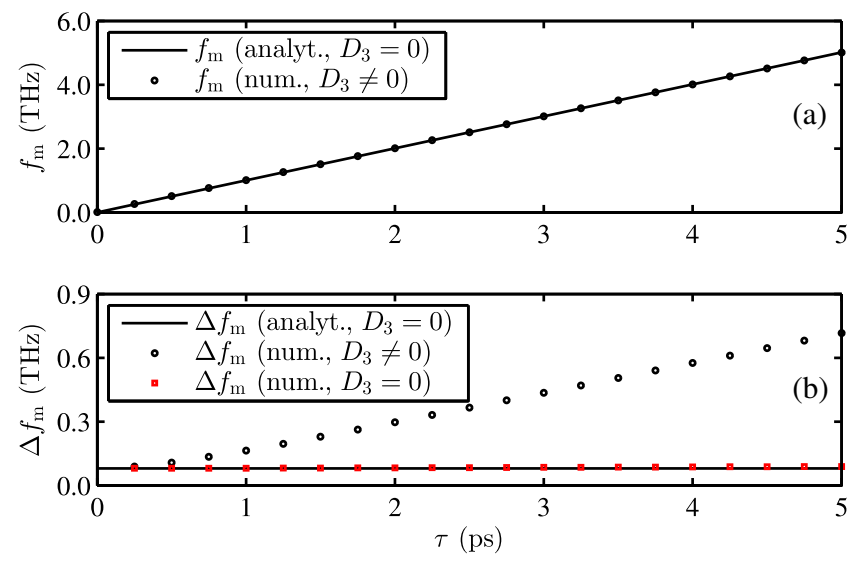

FIG. 3. Calculated modulation frequency $f_{\mathrm{m}}$ (a) and bandwidth $\Delta f_{\mathrm{m}}$ (b) as function of the interferometer delay $\tau$ (see text for details).

see Sec. IV B), $g=1500^{-1} \mathrm{~mm}$, and $\gamma=51.3^{\circ}$ (actual values from the compressor installed at the DELTA shortpulse facility, see Sec. IVA). The GDD $D_{2}=0.159 \mathrm{ps}^{2}$ is calculated via Eq. (4), while $g$ and $\gamma$ are used to calculate $D_{3}=-5.43 \times 10^{-5} \mathrm{ps}^{3}$ according to Eq. (20).

The third-order term $D_{3} \neq 0$ neither affects the pulse length after compression or stretching, nor does it change the linear dependence of the modulation frequency $f_{\mathrm{m}}$ on $\tau$ [Fig. 3(a)]. However, the modulation bandwidth $\Delta f_{\mathrm{m}}$ increases with an asymptotically linear dependence on $\tau$ [Fig. 3(b)], which is expected following Eq. (22).

In order to reduce third-order dispersion for a pulse length of, e.g., $11 \mathrm{ps,}$, an external compressor with different gratings and angles may be designed and placed between the Ti:sapphire amplifier and the Michelson interferometer [see Fig. 1(a)]. Based on the parameters of the internal compressor (see above) at full compression, $\Delta t_{\min } \approx 40 \mathrm{fs}$ and $d \approx 0.265 \mathrm{~m}$, it causes a GDD of $D_{2}=-1.45 \mathrm{ps}^{2}$ and a third-order dispersion of $D_{3}=4.95 \times 10^{-4} \mathrm{ps}^{3}$. Assuming $D_{2}=D_{3}=0$ for the fully compressed pulse, a bypass of the internal compressor would yield an uncompressed pulse with a GDD of $D_{2}=1.45 \mathrm{ps}^{2}$, a third-order dispersion of $D_{3}=-4.95 \times 10^{-4} \mathrm{ps}^{3}$, and a pulse length of 100 ps. With a parameter set of, e.g., $d=0.2027 \mathrm{~m}, \gamma=44^{\circ}$, and $g=1500^{-1} \mathrm{~mm}$, an external compressor would then lead to a pulse length of $11 \mathrm{ps}$ with a final third-order dispersion of $D_{3} \approx 0$ and, therefore, no broadening of the THz spectra [Fig. 3(b)]. If a tuning of the pulse length is desired while keeping $D_{3}=0$, a different compressor type offering independent control of both, second- and third-order dispersion may be set up [39].

\section{Start-to-end simulation}

The process of laser-induced CSR generation has been modeled from start to end using the code $\mathrm{THzSMe}$ [40]: Based on the optical functions of the storage ring, a random 
electron distribution is generated and tracked stepwise along the sinusoidal path in the modulator. In each step, the work on the electrons provided by the laser pulse is calculated (multiplication of the transverse velocity vector with the temporal step width, the electron charge, and the electrical field of the laser pulse). The laser pulse is described by Gaussian beam optics and includes an intensity modulation based on chirped-pulse beating with optional linear chirp $\alpha$ [Eq. (22)]. The electron distribution is then tracked through the storage ring using the code ELEGANT [38]. Finally, the form factor for coherent emission at the $\mathrm{THz}$ beamline is calculated based on a histogram of the longitudinal electron distribution and its Fourier transformation [Eq. (13)].

Figure 4 shows the form factor resulting from a start-toend simulation based on the electron bunch and laser pulse parameters mentioned above (see Secs. III A and III B), which represent the experimental setup at DELTA. The modulation frequency was chosen to be $f_{\mathrm{m}}=1 \mathrm{THz}$. Neglecting third-order dispersion and, thus, a chirp of the intensity modulation $\left(D_{3}=0, \alpha=0\right)$, a bandwidth of $58 \mathrm{GHz}$ (FWHM) is obtained (Fig. 4). Using Eq. (9), the bandwidth for the intensity modulation of the laser pulse should amount to $\Delta f_{\mathrm{m}}=80 \mathrm{GHz}$ (FWHM). Assuming a linear dependence between laser intensity modulation and electron density modulation, a factor of $1 / \sqrt{2}$ in spectral width has to be included, as the form factor $g^{2}(\nu)$ is given by the squared Fourier transform of the density modulation. Thus, a theoretical bandwidth of $\Delta \nu_{\mathrm{THz}}=\Delta f_{\mathrm{m}} / \sqrt{2}=$ $57 \mathrm{GHz}$ is obtained, which is in good agreement with the numerical simulation result of $58 \mathrm{GHz}$.

Figure 4 also shows the form factor resulting from a second simulation with a chirp parameter $\alpha=142 \mathrm{ps}^{-3}$, which was calculated based on a third-order dispersion of $D_{3}=-5.43 \times 10^{-5} \mathrm{ps}^{3}$ (see Sec. III B). According to Fig. 3(b), the intensity modulation bandwidth should increase from $80 \mathrm{GHz}$ to $164 \mathrm{GHz}$. Including the factor of $1 / \sqrt{2}$, a theoretical $\mathrm{THz}$ bandwidth of $164 / \sqrt{2} \mathrm{GHz}=$ $116 \mathrm{GHz}$ is obtained, again in good agreement with the numerical result of $113 \mathrm{GHz}$ (Fig. 4). This increase in spectral width from about $60 \mathrm{GHz}$ to $115 \mathrm{GHz}$ emphasizes the importance of a third-order dispersion compensation using an external compressor.

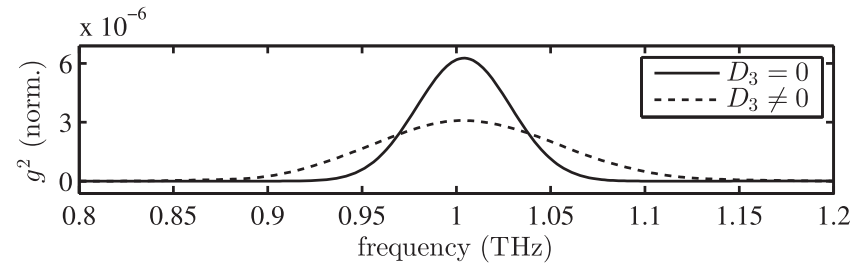

FIG. 4. Narrowband form factors resulting from start-to-end simulations with and without third-order dispersion $D_{3}$ (see text for details).
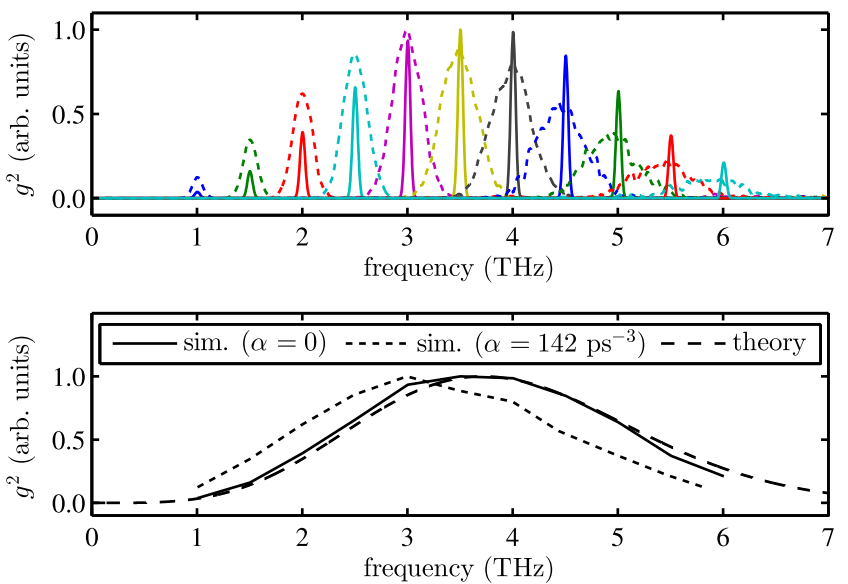

FIG. 5. Top: narrowband form factors resulting from start-toend simulations with (dotted) and without third-order dispersion (solid) for different modulation frequencies (see text for details). Bottom: gain curves extracted from the simulated form factors and according to theory (see Fig. 2).

The start-to-end simulations with and without thirdorder dispersion were repeated for different modulation frequencies, and the resulting form factors and extracted gain curves are shown in Fig. 5 (both sets of data were scaled to provide the same maximum form factor). The theoretical gain curve matches the simulation without third-order dispersion $(\alpha=0)$ well, which supports the validity of the assumptions discussed in Sec. II D. The third-order dispersion then causes a shift of the extracted gain curve towards lower frequencies due to the increased spectral broadening for higher modulation frequencies [see Fig. 3(b)].

\section{EXPERIMENTAL SETUP}

\section{A. DELTA and its short-pulse facility}

DELTA is a synchrotron light source operated by the Center for Synchrotron Radiation of the TU Dortmund University in Dortmund, Germany. Following a $75-\mathrm{MeV}$ linear accelerator, a slow-ramping booster synchrotron allows for full-energy injection into the $1.5-\mathrm{GeV}$ electron storage ring DELTA (Fig. 6). Key parameters of the storage ring are given in Table II.

Since 2011, a short-pulse facility for ultrashort and coherent VUV and $\mathrm{THz}$ pulses based on the coherent harmonic generation $(\mathrm{CHG})$ principle has been established at DELTA $[18,41,42]$.

The 800-nm Ti:sapphire seed-laser system at the beginning of beamline BL3 (Fig. 6) comprises a mode-locked oscillator and a chirped-pulse amplifier with a regenerative multipass and a following single-pass stage. Up to $8 \mathrm{~mJ}$ per pulse are provided at a repetition rate of $1 \mathrm{kHz}$, allowing a laser-electron overlap with a single bunch in the storage ring at every 2600th revolution. The minimum pulse length is about $40 \mathrm{fs}$. 


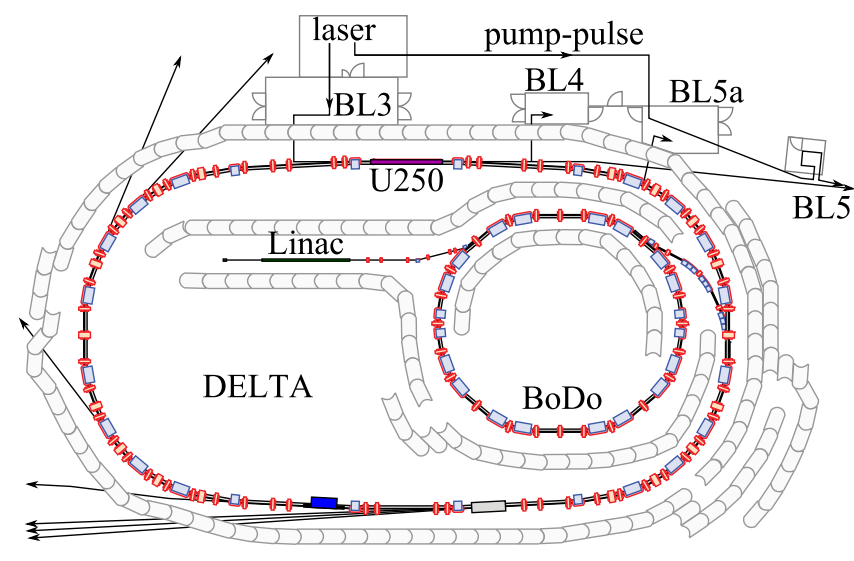

FIG. 6. Layout of DELTA and the short-pulse facility comprising a linear accelerator ("Linac"), a booster synchrotron ("BoDo"), the electron storage ring, the laser laboratory, the seeding beamline BL3, the electromagnetic undulator U250, the diagnostics beamline BL4, the THz beamline BL5a, the VUV beamline BL5, and the pump-pulse beamline (see text for details).

A refractive telescope with three adjustable lenses is used to focus the laser pulses into the electromagnetic undulator U250 (Fig. 6, period length $250 \mathrm{~mm}$ ), which can be tuned to $800 \mathrm{~nm}$ at full beam energy. Following the laser-electron interaction in a first seven-period section (modulator), a magnetic chicane at the center of the U250 transforms the induced energy modulation into a periodic longitudinal density modulation, i.e., a series of microbunches with a distance of the laser wavelength. A third section of the U250 serves as a radiator, in which ultrashort pulses are emitted coherently at harmonics of the laser wavelength. The in-air diagnostics beamline BL4 is used to find and optimize the longitudinal and transverse laser-electron overlap as well as to study the CHG pulses down to a wavelength of $200 \mathrm{~nm}$. The evacuated beamline BL5 is used for CHG studies at shorter wavelengths, and it is planned to perform time-resolved pump-probe experiments employing the VUV pulses from the radiator as probe and a fraction of each seed laser pulse as pump, which is sent directly from the laser laboratory to the BL5 endstation via a separate pump-pulse beamline.

Further downstream, a bending magnet serves as the source for the evacuated $\mathrm{THz}$ beamline BL5a (Fig. 6)

TABLE II. Parameters of the electron storage ring DELTA. nominal beam energy

circumference

revolution time

multibunch current

single-bunch current

bunch length

horizontal beam emittance

relative energy spread

momentum compaction factor
$1.5 \mathrm{GeV}$

$115.2 \mathrm{~m}$

$384 \mathrm{~ns}$

$130 \mathrm{~mA}$ (max.)

$20 \mathrm{~mA}$ (max.)

100 ps (FWHM)

$15 \mathrm{~nm} \mathrm{rad}$

$6.8 \times 10^{-4}$

$5 \times 10^{-3}$
$[40,43,44]$, which comprises several in-air time-resolving detectors and an evacuated Fourier-transform infrared spectrometer with a liquid-helium-cooled silicon composite bolometer. During CHG operation, the sub-picosecond dip in the longitudinal electron density caused by the energy modulation with a 40-fs laser pulse gives rise to broadband pulses in the range of 1 to $5 \mathrm{THz}[26,40,45]$.

\section{B. Chirped-pulse-beating setup}

For the generation of narrowband $\mathrm{THz}$ pulses at DELTA, a temporary chirped-pulse-beating setup [Fig. 1(a)] was installed at the seed-laser beamline BL3 (Fig. 6). With the internal compressor of the Ti:sapphire amplifier detuned to deliver pulses with a length of roughly 11 ps (FWHM), a Michelson interferometer comprising a beam splitter and two corner-cube retroreflectors (one fixed, one on a remotely controlled linear stage) was used to induce an intensity modulation with variable frequency. An external compressor to compensate the third-order dispersion was not available for this first test. Due to the Michelson interferometer and limited reflectivity of the optical components, about $3.5 \mathrm{~mJ}$ out of $8 \mathrm{~mJ}$ available per pulse were sent into the storage ring. To monitor the pulse length and modulation frequency, $1 \%$ of the power was split off and sent into an optical intensity autocorrelator. Both, the interferometer and the autocorrelator, were provided by the Laboratoire de Physique des Lasers, Atomes et Molécules (PhLAM), Lille, resulting in a similar configuration as employed during previous experiments at UVSOR, Japan [29].

The narrowband $\mathrm{THz}$ pulses were studied at the $\mathrm{THz}$ beamline BL5a using the Fourier-transform infrared spectrometer and the silicon composite bolometer. For a background-free detection, a lock-in amplifier locked to the $1-\mathrm{kHz}$ repetition rate of the laser system was placed between the bolometer and readout electronics of the spectrometer. To calculate the squared form factor $g^{2}(\nu)$, the acquired spectra were divided by a separately measured spectrum of the incoherent $\mathrm{THz}$ radiation. Due to a lowfrequency cutoff in the transmission of the Mylar-multilayer beam splitter employed in the spectrometer, spectral components below about $1.2 \mathrm{THz}$ could not be detected.

\section{EXPERIMENTAL RESULTS}

An example set of measured form factors is shown in Fig. 7 (top). With an average laser pulse length of $11.7 \mathrm{ps}$ (FWHM) for this data set, the delay in the Michelson interferometer was varied to produce intensity modulation frequencies between 1.2 and $5.6 \mathrm{THz}$. These modulation frequencies (dashed vertical lines) were measured by the autocorrelator and coincide with the central frequencies of the corresponding form factors of the coherent $\mathrm{THz}$ radiation spectra. With a spectral width (FWHM) of about $210 \mathrm{GHz}$ at a central frequency of $2 \mathrm{THz}$, the form factor 

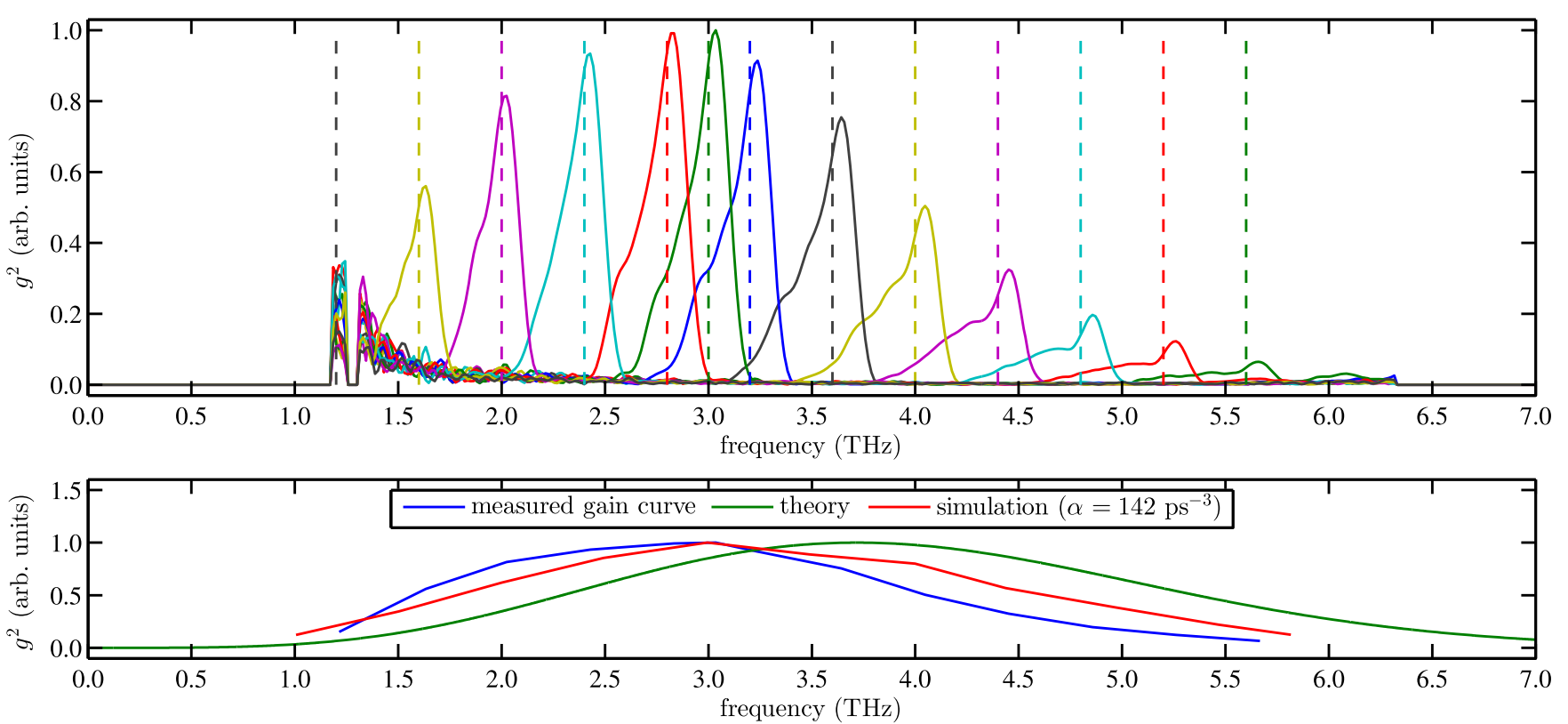

FIG. 7. Top: measured narrowband $\mathrm{THz}$ form factors (solid lines) and corresponding laser modulation frequencies (dashed, see text for details). Bottom: gain curves extracted from the measured (top) and simulated form factors (see Fig. 5) and according to theory (see Fig. 2).

is much narrower than a typical form factor with about $1.5 \mathrm{THz}$ spectral width when seeding with an ultrashort laser pulse [40]. Resulting from the different dispersive properties of the DELTA storage ring, continuously tunable narrowband CSR was generated for the first time at up to $5.6 \mathrm{THz}$, which is an increase in frequency by a factor of about 10 compared to previous similar experiments.

\section{A. Achieved parameters of the THz pulses}

An overview of the parameters for the seed laser and the narrowband $\mathrm{THz}$ pulses resulting from this demonstration experiment is given in Table III. The laser pulse energy and repetition rate were limited by the available laser system. The laser pulse length was chosen as a compromise between the resulting bandwidth-a longer pulse allows for more periods of the intensity modulation and, thus, a more narrow $\mathrm{THz}$ spectrum - and the energy modulation amplitude, which is given by the peak power of the laser pulse. As described by the gain curve (Fig. 7, bottom), the tuning range of the generated $\mathrm{THz}$ pulses is primarily

TABLE III. Parameters of the seed laser and narrowband $\mathrm{THz}$ pulses generated at DELTA. See text for details.

\begin{tabular}{lc}
\hline \hline central laser wavelength & $800 \mathrm{~nm}$ \\
laser pulse energy & $3.5 \mathrm{~mJ}$ \\
laser pulse duration & $11.7 \mathrm{ps}(\mathrm{FWHM})$ \\
repetition rate & $1 \mathrm{kHz}$ \\
THz tuning range & 1.2 to $5.6 \mathrm{THz}$ \\
$\mathrm{THz}$ bandwidth & 190 to $340 \mathrm{GHz}(\mathrm{FWHM})$ \\
THz pulse energy & $\approx 0.1$ to $1 \mathrm{~nJ}$ at 5 to $15 \mathrm{~mA}$ \\
\hline \hline
\end{tabular}

limited by the dispersive properties of the storage ring lattice. Pulses at $1 \mathrm{THz}$ and slightly below from turn 0 as well as pulses at several 10 to $100 \mathrm{GHz}$ emitted after one or more revolutions of the modulated electron bunch in the storage ring (Fig. 2) were generated and could be detected by a $\mathrm{YBa}_{2} \mathrm{Cu}_{3} \mathrm{O}_{7}$ detector $[45,46]$, but their spectra could not be recorded due to the limitations of the employed spectrometer (Sec. IV B). The bandwidth is given by the laser pulse length and the effect of higher-order dispersion (see below). The value for the THz pulse energy in Table III was not measured directly, as a calibrated detector or power meter was not available. Instead, the pulse energy was estimated based on the value for broadband $\mathrm{THz}$ pulses [40] and a comparison of the absolute signal intensity for broad- and narrowband pulses on the same spectrometer as well as on a simulation with $\mathrm{THzSMe}$ (Sec. III C).

\section{B. Discussion of pulse properties}

The increase of the spectral width with increasing central frequency is in general agreement with numerical predictions for the case of third-order dispersion in the laser pulse [Fig. 3(b)]. Considering the data shown in Fig. 7, the spectral width increases from about $190 \mathrm{GHz}$ at $1.6 \mathrm{THz}$ to $340 \mathrm{GHz}$ at $5.6 \mathrm{THz}$. With the numerical simulation [Fig. 3(b)] predicting bandwidths of about 170 to $560 \mathrm{GHz}$ (including the factor of $\sqrt{1 / 2}$ discussed in Sec. III C), the amount of positive third-order dispersion is somewhat smaller in the measurement as compared to the numerical prediction. This smaller positive third-order dispersion in the laser pulse after passing the detuned compressor suggests a negative amount of third-order dispersion at 


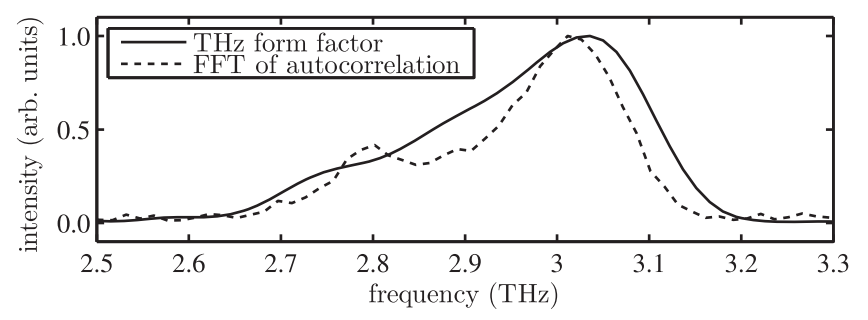

FIG. 8. Narrowband 3-THz form factor (also shown in Fig. 7) and fast Fourier transform (FFT) of the corresponding laser pulse autocorrelation trace.

full compression, which was confirmed later employing a FROG device [47].

The form factors in Fig. 7 (top) show a non-Gaussian shape with low-frequency tails. Comparing the measured form factor at $3 \mathrm{THz}$ central frequency to a Fourier transform of the corresponding autocorrelation data (Fig. 8), this asymmetric spectral shape is found to be already present in the modulated laser pulse and might be related to fourth-order dispersion or spectral distortions caused by the optical components in the beam path.

A gain curve extracted from the peak intensities of all individual form factors is shown in the bottom half of Fig. 7 (blue). Contrary to the theoretical curve according to Eq. (18) (green), the simulated curve (red) based on a quadratic chirp parameter of $\alpha=142 \mathrm{ps}^{-3}$ (see Fig. 5) shows a closer match to the experimental curve. This indicates that the primary effect for the shift of the gain curve toward lower frequencies is the third-order dispersion. The remaining discrepancy between simulation and experiment is assumed to be caused by a different amount of third-order dispersion (see above) and other small deviations of the simulation parameters, e.g., a limited mismatch between the simulated and actual storage ring optics [40].

\section{Comparison to other sources and options for improvement}

Alternative sources for coherent narrowband pulses with continuously tunable frequencies in the multi-THz regime are far-infrared or $\mathrm{THz}$ free-electron lasers (FELs) as well as superradiant $\mathrm{THz}$ undulator sources, at which ultrashort electron bunches emit coherent multicycle $\mathrm{THz}$ pulses while passing an undulator tuned to the desired frequency. In recent years, pulse energies up to $100 \mu \mathrm{J}$ have been generated at repetition rates of a few $10 \mathrm{~Hz}[48,49]$. Pulse energies up to $1 \mu \mathrm{J}$ have been produced even at a few $100 \mathrm{kHz}$ using superconducting accelerator technology, and an increase to several $100 \mu \mathrm{J}$ at up to a few $\mathrm{MHz}$ is expected [50]. Another alternative are laser-based table-top $\mathrm{THz}$ sources such as photoconductive antennas [51] or optical rectification [52]. Combined with a method like chirped-pulse beating for intensity-modulated laser pulses, tunable narrowband pulses with up to $1 \mu \mathrm{J}$ at $1 \mathrm{kHz}$ or $10 \mu \mathrm{J}$ at $10 \mathrm{~Hz}$ have been generated in the $\mathrm{THz}$ regime [53].

The results shown in this article represent a first demonstration experiment for narrowband CSR from a storage ring with continuously tunable frequencies in the multi-THz regime. Although it is doubtful that this method will reach the pulse energies and repetition rates of a dedicated superconducting $\mathrm{THz}$ FEL or superradiant $\mathrm{THz}$ undulator source, there are several options to improve the performance. Employing a different laser system, the repetition rate and/or laser pulse energy may be increased. A higher pulse energy would in turn allow for a stronger electron energy modulation leading to increased $\mathrm{THz}$ pulse energies, or for a longer laser pulse with more modulation periods leading to a decreased bandwidth and increased number of modulated electrons. Compensating third-order dispersion (Sec. III B) would also decrease the bandwidth significantly, allowing for a higher peak power at a given frequency. Finally, the $\mathrm{THz}$ pulse energy would benefit from increased electron densities, which may be achieved by higher bunch currents or reduced bunch lengths as they are available at several electron storage rings. With these improvements, the achievable parameters might be comparable to those of modern table-top sources, which in turn suffer from a limited tunability, e.g., due to two-phonon absorption in the antenna substrate or nonlinear crystal [49]. Compared to the effort of establishing a new dedicated superconducting linear accelerator serving a THz FEL or superradiant $\mathrm{THz}$ undulator source, the presented method can easily be implemented at several existing synchrotron radiation facilities broadening the scope for user applications. Thus, it may prove to be an alternative to the limited number of THz FELs or superradiant $\mathrm{THz}$ undulator sources when extreme pulse energies or field gradients are not mandatory.

\section{CONCLUSION AND OUTLOOK}

The method of chirped-pulse beating allows for the generation of coherent narrowband $\mathrm{THz}$ radiation pulses with continuously tunable frequencies and can easily be implemented at storage rings already employing a laser-induced energy modulation. At DELTA, narrowband THz pulses were generated at central frequencies up to $5.6 \mathrm{THz}$, extending the accessible frequency range from previous similar experiments significantly and almost closing the "THz gap". A first application of this source has been the study of the frequency-dependent radiation detection mechanism in thin-film $\mathrm{YBa}_{2} \mathrm{Cu}_{3} \mathrm{O}_{7}[45,46]$. The significant effect of third-order dispersion - broadened $\mathrm{THz}$ spectra with reduced peak intensity especially at higher modulation frequencies - and further deviations of the spectral shape emphasize the importance of a more detailed knowledge and control of the laser pulse properties. Parameters for an external pulse compressor to reduce third-order dispersion have been suggested.

A permanent chirped-pulse-beating setup for DELTA incorporating an optimized compressor is currently under 
construction and will allow us to generate, study, and provide tunable narrowband $\mathrm{THz}$ pulses for user experiments on a more regular basis. Employing a Martin-Puplett spectrometer which was recently commissioned [27], it will also be possible to study sub-THz spectra emitted after one or more revolutions of the modulated electron bunch in the storage ring.

\section{ACKNOWLEDGMENTS}

It is a pleasure to thank our colleagues at DELTA and the Faculty of Physics at the TU Dortmund University for their continuous support. Furthermore, the project has profited from the expertise of our colleagues at UVSOR (Institute for Molecular Science, Okazaki, Japan), ANKA/IPS/LAS and IMS (Karlsruhe Institute of Technology, Karlsruhe, Germany), BESSY (Helmholtz-Zentrum Berlin, Berlin, Germany), MLS (Physikalisch-Technische Bundesanstalt, Berlin, Germany), DESY (Hamburg, Germany), and SLS (Paul Scherrer Institute, Villigen, Switzerland). The work at DELTA was funded by the German Federal Ministry of Education and Research (BMBF) under Contracts No. 05K10PEB, No. 05K13PEC, and No. 05K16PEB, by the German Research Foundation (DFG) under Contract No. INST 212/236-1 FUGG, by the German Federal State of North Rhine-Westphalia (NRW), and by the TU Dortmund University. The PhLAM acknowledges the support from the French Ministry of Higher Education and Research (MESR), the Nord-Pas de Calais Regional Council, and the European Regional Development Fund (ERDF) through the Contrat de plan État-région Photonics for Society (CPER P4s), and from the French National Research Agency (ANR) through the LabEx CEMPI project (ANR-11-LABX-0007).

[1] J. Feikes, M. von Hartrott, M. Ries, P. Schmid, G. Wüstefeld, A. Hoehl, R. Klein, R. Müller, and G. Ulm, Metrology Light Source: The first electron storage ring optimized for generating coherent $\mathrm{THz}$ radiation, Phys. Rev. ST Accel. Beams 14, 030705 (2011).

[2] E. Roussel, C. Evain, C. Szwaj, S. Bielawski, J. Raasch, P. Thoma, A. Scheuring, M. Hofherr, K. Ilin, S. Wünsch, M. Siegel, M. Hosaka, N. Yamamoto, Y. Takashima, H. Zen, T. Konomi, M. Adachi, S. Kimura, and M. Katoh, Microbunching Instability in Relativistic Electron Bunches: Direct Observations of the Microstructures Using Ultrafast YBCO Detectors, Phys. Rev. Lett. 113, 094801 (2014).

[3] A. Schnegg, J. Behrends, K. Lips, R. Bittl, and K. Holldack, Frequency domain Fourier transform THz-EPR on single molecule magnets using coherent synchrotron radiation, Phys. Chem. Chem. Phys. 11, 6820 (2009).

[4] P. Probst, A. Semenov, M. Ries, A. Hoehl, P. Rieger, A. Scheuring, V. Judin, S. Wünsch, K. Il'in, N. Smale, Y.-L. Mathis, R. Müller, G. Ulm, G. Wüstefeld, H.-W. Hübers, J. Hänisch, B. Holzapfel, M. Siegel, and A.-S. Müller,
Nonthermal response of $\mathrm{YBa}_{2} \mathrm{Cu}_{3} \mathrm{O}_{7-\delta}$ thin films to picosecond THz pulses, Phys. Rev. B 85, 174511 (2012).

[5] J. Barros, C. Evain, E. Roussel, L. Manceron, J.-B. Brubach, M.-A. Tordeux, M.-E. Couprie, S. Bielawski, C. Szwaj, M. Labat, and P. Roy, Characteristics and development of the coherent synchrotron radiation sources for THz spectroscopy, J. Mol. Spectrosc. 315, 3 (2015).

[6] V. Bragaglia, A. Schnegg, R. Calarco, and K. Holldack, Epitaxial $\mathrm{Ge}_{2} \mathrm{Sb}_{2} \mathrm{Te}_{5}$ probed by single cycle $\mathrm{THz}$ pulses of coherent synchrotron radiation, Appl. Phys. Lett. 109, 141903 (2016).

[7] A. Andersson, M. S. Johnson, and B. Nelander, Coherent synchrotron radiation in the far infrared from a $1-\mathrm{mm}$ electron bunch, Opt. Eng. 39, 3099 (2000).

[8] G. Carr, S. Kramer, J. Murphy, R. Lobo, and D. Tanner, Observation of coherent synchrotron radiation from the NSLS VUV ring, Nucl. Instrum. Methods Phys. Res., Sect. A 463, 387 (2001).

[9] U. Arp, G. T. Fraser, A. R. Hight Walker, T. B. Lucatorto, K. K. Lehmann, K. Harkay, N. Sereno, and K.-J. Kim, Spontaneous coherent microwave emission and the sawtooth instability in a compact storage ring, Phys. Rev. ST Accel. Beams 4, 054401 (2001).

[10] J. M. Byrd, W. P. Leemans, A. Loftsdottir, B. Marcelis, M. C. Martin, W. R. McKinney, F. Sannibale, T. Scarvie, and C. Steier, Observation of Broadband Self-Amplified Spontaneous Coherent Terahertz Synchrotron Radiation in a Storage Ring, Phys. Rev. Lett. 89, 224801 (2002).

[11] G. Stupakov and S. Heifets, Beam instability and microbunching due to coherent synchrotron radiation, Phys. Rev. ST Accel. Beams 5, 054402 (2002).

[12] M. Venturini and R. Warnock, Bursts of Coherent Synchrotron Radiation in Electron Storage Rings: A Dynamical Model, Phys. Rev. Lett. 89, 224802 (2002).

[13] M. Abo-Bakr, J. Feikes, K. Holldack, G. Wüstefeld, and H.-W. Hübers, Steady-State Far-Infrared Coherent Synchrotron Radiation detected at BESSY II, Phys. Rev. Lett. 88, 254801 (2002).

[14] S. Khan, K. Holldack, T. Kachel, R. Mitzner, and T. Quast, Femtosecond Undulator Radiation from Sliced Electron Bunches, Phys. Rev. Lett. 97, 074801 (2006).

[15] G. Ingold, P. Beaud, S. L. Johnson, D. Grolimund, V. Schlott, T. Schmidt, and A. Streun, Technical Report: FEMTO: A Sub-ps Tunable Hard X-ray Undulator Source for Laser/X-ray Pump-Probe Experiments at the SLS, Synchrotron Radiat. News 20, 35 (2007).

[16] G. De Ninno, E. Allaria, M. Coreno, F. Curbis, M. B. Danailov, E. Karantzoulis, A. Locatelli, T. O. Menteş, M. A. Nino, C. Spezzani, and M. Trovò, Generation of Ultrashort Coherent Vacuum Ultraviolet Pulses Using Electron Storage Rings: A New Bright Light Source for Experiments, Phys. Rev. Lett. 101, 053902 (2008).

[17] M. Labat, G. Lambert, M. Couprie, M. Shimada, M. Katoh, M. Hosaka, Y. Takashima, T. Hara, and A. Mochihashi, Coherent Harmonic Generation experiments on UVSOR-II storage ring, Nucl. Instrum. Methods Phys. Res., Sect. A 593, 1 (2008).

[18] S. Khan, M. Bakr, M. Höner, H. Huck, R. Molo, A. Nowaczyk, A. Schick, P. Ungelenk, and M. Zeinalzadeh, Coherent Harmonic Generation at DELTA: A New Facility 
for Ultrashort Pulses in the VUV and $\mathrm{THz}$ Regime, Synchrotron Radiat. News 24, 18 (2011).

[19] M. Labat et al., Commissioning progress of the Femto-slicing Project at SOLEIL, in Proceedings of the 5th International Particle Accelerator Conference IPAC 2014, Dresden, Germany (JACoW, Geneva, 2014), p. 206, http://accelconf.web.cern.ch/AccelConf/IPAC2014/papers/ mopro054.pdf.

[20] A. A. Zholents and M. S. Zolotorev, Femtosecond X-Ray Pulses of Synchrotron Radiation, Phys. Rev. Lett. 76, 912 (1996).

[21] R. Coisson and F. de Martini, Free-Electron Coherent Relativistic Scatterer for UV Generation, in Free-Electron Generators of Coherent Radiation, Physics of Quantum Electronics (Addison-Wesley Publishing Company, Massachusetts, 1982), Vol. 9.

[22] R. Prazeres, J. Ortega, C. Bazin, M. Bergher, M. Billardon, M. Couprie, M. Velghe, and Y. Petroff, Coherent harmonic generation in the vacuum ultraviolet spectral range on the storage ring ACO, Nucl. Instrum. Methods Phys. Res., Sect. A 272, 68 (1988).

[23] K. Holldack, S. Khan, R. Mitzner, and T. Quast, Femtosecond Terahertz Radiation from Femtoslicing at BESSY, Phys. Rev. Lett. 96, 054801 (2006).

[24] J. M. Byrd, Z. Hao, M. C. Martin, D. S. Robin, F. Sannibale, R. W. Schoenlein, A. A. Zholents, and M.S. Zolotorev, Tailored Terahertz Pulses from a Laser-Modulated Electron Beam, Phys. Rev. Lett. 96, 164801 (2006).

[25] M. Shimada, M. Katoh, S. ichi Kimura, A. Mochihashi, M. Hosaka, Y. Takashima, T. Hara, and T. Takahashi, Intense Terahertz Synchrotron Radiation by Laser Bunch Slicing at UVSOR-II Electron Storage Ring, Jpn. J. Appl. Phys. 46, 7939 (2007).

[26] P. Ungelenk, H. Huck, M. Huck, M. Höner, S. Khan, R. Molo, A. Schick, N. Hiller, and V. Judin, Temporal and spectral observation of laser-induced $\mathrm{THz}$ radiation at DELTA, in Proceedings of the 4th International Particle Accelerator Conference IPAC 2013, Shanghai, China (JACoW, Geneva, 2013), p. 94, http://accelconf.web.cern .ch/AccelConf/IPAC2013/papers/mopea014.pdf.

[27] C. Mai, P. Ungelenk, F. Bahnsen, M. Bolsinger, S. Hilbrich, M. Huck, M. Höner, S. Khan, A. M. auf der Heide, R. Molo, H. Rast, G. Shayeganrad, M. Brosi, B. Kehrer, A.-S. Müller, M. Nasse, P. Schönfeldt, P. Schütze, S. Walther, and H. Huck, Observation of coherent pulses in the sub-THz range at DELTA, in Proceedings of the 6th International Particle Accelerator Conference IPAC 2015, Richmond, USA (JACoW, Geneva, 2015), p. 823, http://accelconf.web.cern .ch/AccelConf/IPAC2015/papers/mopha023.pdf.

[28] C. Mai, F. Bahnsen, M. Bolsinger, F. Götz, S. Hilbrich, M. Höner, M. Jebramcik, S. Khan, N. Lockmann, A. M. auf der Heide, R. Molo, R. Niemczyk, G. Shayeganrad, M. Suski, P. Ungelenk, and D. Zimmermann, Time-resolved spectral observation of coherent THz pulses at DELTA, in Proceedings of the 7th International Particle Accelerator Conference IPAC 2016, Busan, Korea (JACoW, Geneva, 2016), p. 105, http://accelconf.web.cern.ch/AccelConf/ ipac2016/papers/mopmb013.pdf.

[29] S. Bielawski, C. Evain, T. Hara, M. Hosaka, M. Katoh, S. Kimura, A. Mochihashi, M. Shimada, C. Szwaj,
T. Takahashi, and Y. Takashima, Tunable narrowband terahertz emission from mastered laser-electron beam interaction, Nat. Phys. 4, 390 (2008).

[30] A. S. Weling, B. B. Hu, N. M. Froberg, and D. H. Auston, Generation of tunable narrow-band $\mathrm{THz}$ radiation from large aperture photoconducting antennas, Appl. Phys. Lett. 64, 137 (1994).

[31] A. S. Weling and D. H. Auston, Novel sources and detectors for coherent tunable narrow-band terahertz radiation in free space, J. Opt. Soc. Am. B 13, 2783 (1996).

[32] C. Evain, C. Szwaj, S. Bielawski, M. Hosaka, Y. Takashima, M. Shimada, S. Kimura, M. Katoh, A. Mochihashi, T. Takahashi, and T. Hara, Laser-induced narrowband coherent synchrotron radiation: Efficiency versus frequency and laser power, Phys. Rev. ST Accel. Beams 13, 090703 (2010).

[33] D. Strickland and G. Mourou, Compression of amplified chirped optical pulses, Opt. Commun. 55, 447 (1985).

[34] L. I. Schiff, Production of Particle Energies beyond $200 \mathrm{Mev}$, Rev. Sci. Instrum. 17, 6 (1946).

[35] H. Wiedemann, Particle Accelerator Physics, 4th ed. (Springer, New York, 2015), p. 847.

[36] E. Treacy, Optical pulse compression with diffraction gratings, IEEE J. Quantum Electron. 5, 454 (1969).

[37] I. Christov and I. Tomov, Large bandwidth pulse compression with diffraction gratings, Opt. Commun. 58, 338 (1986).

[38] M. Borland, elegant: A Flexible SDDS-Compliant Code for Accelerator Simulation, Advanced Photon Source LS-287 (Argonne National Laboratory, Argonne, 2000).

[39] V. Chauhan, P. Bowlan, J. Cohen, and R. Trebino, Single-diffraction-grating and grism pulse compressors, J. Opt. Soc. Am. B 27, 619 (2010).

[40] P. Ungelenk, Generation and Detection Schemes for Laser-Induced Coherent Terahertz Radiation at the Electron Storage Ring DELTA, Dissertation, TU Dortmund University, Dortmund, 2015.

[41] H. Huck, M. Bakr, M. Höner, S. Khan, R. Molo, A. Nowaczyk, A. Schick, P. Ungelenk, and M. Zeinalzadeh, Coherent harmonic generation at the DELTA storage ring, in Proceedings of the 33rd International Free Electron Laser Conference FEL 2011, Shanghai, China (JACoW, Geneva, 2011), p. 5, http://accelconf.web.cern .ch/AccelConf/FEL2011/papers/mooa5.pdf.

[42] S. Khan, F. Bahnsen, S. Cramm, S. Döring, J. Grewe, M. Höner, H. Huck, M. Huck, R. Molo, L. Plucinski, A. Schick, C. M. Schneider, and P. Ungelenk, Generation of Ultrashort and Coherent Synchrotron Radiation Pulses at DELTA, Synchrotron Radiat. News 26, 25 (2013).

[43] M. Höner, P. Ungelenk, M. Bakr, H. Huck, S. Khan, R. Molo, A. Nowaczyk, A. Schick, and M. Zeinalzadeh, A dedicated THz beamline at DELTA, in Proceedings of the 2nd International Particle Accelerator Conference IPAC 2011, San Sebastián, Spain (JACoW, Geneva, 2011), p. 2939, http://accelconf.web.cern.ch/AccelConf/ IPAC2011/papers/thpc015.pdf.

[44] P. Ungelenk, M. Bakr, M. Höner, H. Huck, S. Khan, R. Molo, A. Nowaczyk, A. Schick, and M. Zeinalzadeh, Recent developments at the DELTA $\mathrm{THz}$ beamline, in Proceedings of the 3rd International Particle Accelerator 
Conference IPAC 2012, New Orleans, USA (IEEE, Piscataway, NJ, 2012), p. 768, http://accelconf.web.cern .ch/AccelConf/IPAC2012/papers/moppp091.pdf.

[45] P. Ungelenk, L.-G. Böttger, S. Hilbrich, H. Huck, M. Huck, M. Höner, S. Khan, C. Mai, A. Meyer auf der Heide, R. Molo, H. Rast, A. Schick, S. Bielawski, C. Evain, M. L. Parquier, E. Roussel, C. Szwaj, N. Hiller, V. Judin, J. Raasch, and P. Thoma, Studies of ultrashort THz pulses at DELTA, in Proceedings of the 5th International Particle Accelerator Conference IPAC 2014, Dresden, Germany (JACoW, Geneva, 2014), p. 1936, http://accelconf.web .cern.ch/AccelConf/IPAC2014/papers/wepro002.pdf.

[46] J. Raasch, M. Arndt, K. Ilin, A. Kuzmin, M. Siegel, S. Hilbrich, M. Höner, H. Huck, M. Huck, S. Khan, C. Mai, A. Meyer auf der Heide, R. Molo, H. Rast, P. Ungelenk, S. Bielawski, C. Evain, M. L. Parquier, E. Roussel, and C. Szwaj, Tunable narrowband $\mathrm{THz}$ radiation for the investigation of the frequency-dependent picosecond response of YBCO detectors, in Proceedings of the 10th DELTA User Meeting (Center for Synchrotron Radiation (DELTA), Dortmund, 2014), p. 5, http://www.delta.tu-dortmund.de/
cms/Medienpool/User_Reports/DELTA_User_Report_2014 .pdf.

[47] R. Trebino, Frequency-resolved Optical Gating: The Measurement of Ultrashort Laser Pulses (Kluwer Academic, Boston, 2000).

[48] A. F. G van der Meer, FELs, nice toys or efficient tools?, Nucl. Instrum. Methods Phys. Res., Sect. A 528, 8 (2004).

[49] N. Stojanovic and M. Drescher, Accelerator- and laserbased sources of high-field terahertz pulses, J. Phys. B 46, 192001 (2013).

[50] B. Green et al., High-Field High-Repetition-Rate Sources for the Coherent THz Control of Matter, Sci. Rep. 6, 22256 (2016).

[51] D. H. Auston and P. R. Smith, Generation and detection of millimeter waves by picosecond photoconductivity, Appl. Phys. Lett. 43, 631 (1983).

[52] M. Bass, P. A. Franken, J. F. Ward, and G. Weinreich, Optical Rectification, Phys. Rev. Lett. 9, 446 (1962).

[53] Z. Chen, X. Zhou, C. A. Werley, and K. A. Nelson, Generation of high power tunable multicycle teraherz pulses, Appl. Phys. Lett. 99, 071102 (2011). 